\title{
Development of a Reverse Transcription Loop-Mediated Isothermal Amplification Method for the Rapid Detection of Subtype H7N9 Avian Influenza Virus
}

\author{
Hongmei Bao, Yuhui Zhao, Yunhe Wang, Xiaolong Xu, Jianzhong Shi, Xianying Zeng, \\ Xiurong Wang, and Hualan Chen
}

Animal Influenza Laboratory of the Ministry of Agriculture, State Key Laboratory of Veterinary Biotechnology, Harbin
Veterinary Research Institute, Chinese Academy of Agricultural Sciences, 427 Maduan Street, Harbin 150001, China

Correspondence should be addressed to Xiurong Wang; wxr@hvri.ac.cn and Hualan Chen; chenhualan@caas.cn

Received 11 October 2013; Accepted 20 December 2013; Published 6 February 2014

Academic Editor: Roland S. Croner

Copyright (C) 2014 Hongmei Bao et al. This is an open access article distributed under the Creative Commons Attribution License, which permits unrestricted use, distribution, and reproduction in any medium, provided the original work is properly cited.

\begin{abstract}
A novel influenza A (H7N9) virus has emerged in China. To rapidly detect this virus from clinical samples, we developed a reverse transcription loop-mediated isothermal amplification (RT-LAMP) method for the detection of the H7N9 virus. The minimum detection limit of the RT-LAMP assay was 0.01 PFU H7N9 virus, making this method 100-fold more sensitive to the detection of the H7N9 virus than conventional RT-PCR. The H7N9 virus RT-LAMP assays can efficiently detect different sources of H7N9 influenza virus RNA (from chickens, pigeons, the environment, and humans). No cross-reactive amplification with the RNA of other subtype influenza viruses or of other avian respiratory viruses was observed. The assays can effectively detect H7N9 influenza virus RNA in drinking water, soil, cloacal swab, and tracheal swab samples that were collected from live poultry markets, as well as human H7N9 virus, in less than 30 min. These results suggest that the H7N9 virus RT-LAMP assays were efficient, practical, and rapid diagnostic methods for the epidemiological surveillance and diagnosis of influenza A (H7N9) virus from different resource samples.
\end{abstract}

\section{Introduction}

A novel reassortant avian influenza A (H7N9) virus emerged and spread among humans in China in March 2013 [1]. As of August 11, 2013, there have been 135 laboratoryconfirmed human cases in eleven provinces in China, and 44 of these cases resulted in death [2]. Novel H7N9 viruses were simultaneously prevalent in chickens, pigeons, and in the environment of the live poultry markets [3]. The H7N9 virus poses a serious threat to public health [4]. Therefore, the development of a simple and rapid diagnostic method, which can quickly diagnose and timely monitor the H7N9 influenza virus, is extremely important. An accurate diagnosis will allow the timely administration of antiviral therapy and may enable the quarantining of infected cases to prevent the further spread of the virus [5].

Currently, several laboratory techniques for the detection of the H7N9 virus include molecular diagnostics, virus isolation, typing by hemagglutination inhibition and neuraminidase assay, and serology [6-11]. However, the isolation and identification of viruses require extended periods, which range from days to weeks, and do not meet the time requirements that are necessary for the prevention of epidemics. In addition, the H7N9 influenza causes disease among humans and is a significant threat for public health [12]; consequently, the virus must be handled in biosafety level 3 facilities. Therefore, rapid, sensitive, and specific molecular diagnostics have played important roles in the rapid detection of H7N9 influenza viruses. A real-time reverse transcription (RT)-PCR (rRT-PCR) assay has been used to detect H7N9 influenza virus RNA [6]. The realtime RT-PCR is performed in a closed system to minimize contamination. However, rRT-PCR requires an expensive machine system, primers/probes with special modifications, and experienced laboratory workers. 
The loop-mediated isothermal amplification (LAMP) assay is a rapid, accurate, and cost-effective diagnostic method that amplifies the target nucleic acid under isothermal conditions [13]. This method does not require an additional reverse transcription step; only a water bath or heating block is required to amplify large amounts of nucleic acids in 30-60 min [14]. In addition, nucleic acid electrophoresis is not required to assess the results, which can be determined by visualizing a white magnesium pyrophosphate precipitate (easier to be observed after the addition of a fluorescent dye) [15]. Therefore, LAMP is easy to introduce in the frontline or poorly equipped laboratories. In the previous study, we developed the RT-LAMP method for the rapid detection of influenza virus subtype H7 [16]. This RT-LAMP assay has the capacity to detect both high- and low-pathogenic H7 AIV strains. In the present study, we evaluated the ability of H7-RT-LAMP to detect a novel influenza A (H7N9) virus that was identified in humans and birds in China and developed a novel one-step RT-LAMP assay that was specific for the neuraminidase (NA) gene of the H7N9 influenza virus. The H7N9 influenza virus RT-LAMP assays may enable the diagnosis of pandemic H7N9 influenza virus infection faster and more easily from clinical samples of different species.

\section{Materials and Methods}

2.1. Virus Strains. Reference influenza virus subtype strains N1-N9, H7N9 influenza virus subtype (A/chicken/ Shanghai/S1053/2013(H7N9), A/pigeon/Shanghai/S106/2013 (H7N9), A/environment/Shanghai/S1088/2013 (H7N9)), and $\mathrm{A} /$ Anhui/1/2013 (AH/1) $[1,3]$, which were used in the present studies, were maintained and provided by the Animal Influenza Laboratory of the Ministry of Agriculture (Harbin, China) (Table 1). The Newcastle disease virus (NDV), avian infectious bronchitis virus (IBV), and infectious laryngotracheitis virus (ILTV) were provided by the State Key Laboratory of Veterinary Biotechnology, Harbin Veterinary Research Institute (HVRI), Chinese Academy of Agricultural Sciences (CAAS).

2.2. RNA Extraction. Genomic viral RNA was extracted using an RNeasy Mini Kit (Qiagen, Valencia, CA) according to the manufacturer's protocol. RNA isolation from the highly pathogenic avian influenza virus and H7N9 influenza viruses was performed in a biosafety level 3 laboratory at the Harbin Veterinary Research Institute. The RNA concentration $(n g / \mu \mathrm{L})$ was measured with a NanoDrop ND-1000 apparatus (Thermo Scientific).

2.3. H7-RT-LAMP Assay. The H7-RT-LAMP was performed with primers (outer primers (H7-F3 and H7-B3), inner primers (H7-FIP and H7-BIP), and loop primers (H7-LF and H7-LB)) that were specific for the H7 AIV HA gene, as described previously [16].

2.4. H7N9 Influenza Virus NA-Specific RT-LAMP Assay. Primers that were specific for the NA gene of N9 influenza

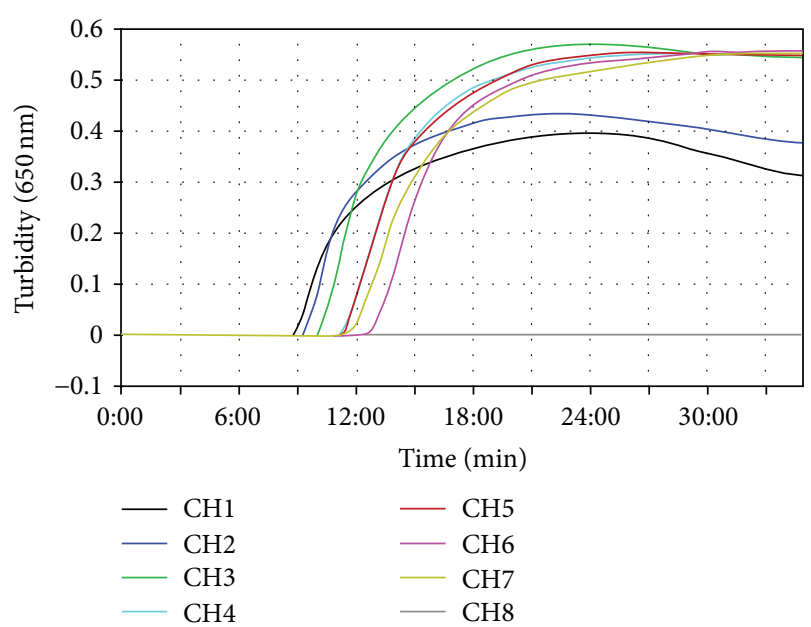

(a)

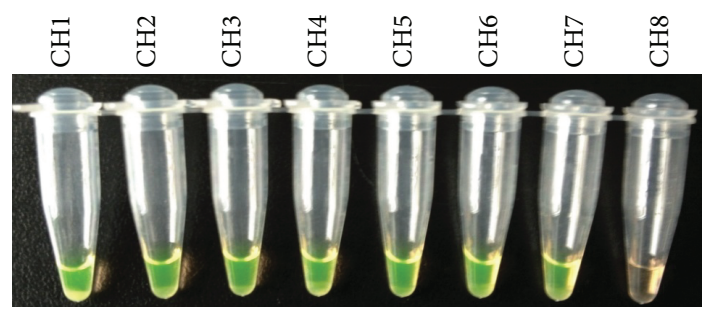

(b)

Figure 1: Detection of H7N9 influenza viruses by H7-RT-LAMP. Viral RNAs were extracted from the H7N9 influenza viruses and were amplified using H7-RT-LAMP. LAMP products were detected by a real-time turbidity assay using an LA-320c (a) and a fluorescence assay (b). CH1: A/CK/Shanghai/S1053/2013 (H7N9); CH2: A/EN/Shanghai/S1088/2013 (H7N9); CH3: A/PG/Shanghai/ S1069/2013 (H7N9); CH4: A/DK/Anhui/SC702/2013 (H7N9); CH5: A/CK/Jiangsu/SC002/2013 (H7N9); CH6: A/Anhui/1/2013 (H7N9); CH7: A/African starling/England/983/79 (H7N1); CH8, negative control.

viruses were designed using the Primer Explorer version 4 software (Eiken Chemical Co., Ltd., Tokyo, Japan; http://primerexplorer.jp/elamp4.0.0/index.html) and synthesized by Shanghai Invitrogen Co., Ltd. A set of six primers included two outer primers (forward primer N9-F3 and reverse primer N9-B3), two inner primers (forward inner primer N9-FIP and reverse inner primer N9-BIP), and two loop primers (forward loop primer N9-LF and reverse loop primer N9-LB) (Figure 1). The RT-LAMP assay was performed in a final reaction volume of $25 \mu \mathrm{L}$, which consisted of the N9-FIP and N9-BIP inner primers $(1.6 \mu \mathrm{M})$, as well as the N9-F3 and N9-B3 outer primers $(0.2 \mu \mathrm{M})$, as described previously (Bao). Amplification reactions were performed from $55^{\circ} \mathrm{C}$ to $65^{\circ} \mathrm{C}$ for $30 \mathrm{~min}$ and at $62.5^{\circ} \mathrm{C}$ for $30 \mathrm{~min}, 45 \mathrm{~min}$, and $60 \mathrm{~min}$ using either an LA-320C Loopamp real-time turbidimeter (Teramecs, Japan) or a water bath. Respective mixtures were heated at $80^{\circ} \mathrm{C}$ for $10 \mathrm{~min}$ to terminate respective reactions. LAMP products were then evaluated by fluorescent detection with a fluorescent detection reagent (Eiken Chemical Co., Ltd.). A positive control (a sample known to be positive for the template) 
TABLE 1: Different subtypes of avian influenza viruses used for validation and specificity of RT-LAMP.

\begin{tabular}{|c|c|c|c|}
\hline Subtype & Virus & Subtype & GenBank accession number \\
\hline $\mathrm{N} 1$ & A/Goose/Guangdong/1/96 & H5N1 & AF144305 \\
\hline $\mathrm{N} 2$ & A/Turkey/Wisconsin/1/66 & H9N2 & D90305 \\
\hline N3 & A/Duck/Germany/1215/73 & $\mathrm{H} 2 \mathrm{~N} 3$ & AY207522 \\
\hline N4 & A/Turkey/Ontario/6118/68 & $\mathrm{H} 8 \mathrm{~N} 4$ & D90305 \\
\hline N5 & A/Mallard/Gurjev/263/82 & H14N5 & M35997 \\
\hline N6 & A/Duck/Czech/56 & H4N6 & D90306 \\
\hline N7 & A/equine/jingfang/1/74 & H7N7 & - \\
\hline N8 & A/Duck/Ukraine/1/63 & $\mathrm{H} 3 \mathrm{~N} 8$ & V01087 \\
\hline N9 & A/chicken/Shanghai/S1053/2013 & H7N9 & CY146958 \\
\hline
\end{tabular}

TABLE 2: Details of RT-LAMP and RT-PCR primers designed for detection of NA gene sequences of N9 subtype influenza viruses.

\begin{tabular}{|c|c|c|c|}
\hline Primer name $^{a}$ & Genome position & Length(s) & Sequences $\left(5^{\prime}\right.$ to $\left.3^{\prime}\right)$ \\
\hline N9-F3 & & 18-mer & GTTTCATGCGACCCAGAT \\
\hline N9-B3 & & 20-mer & CATGGCAACTAGTACTTGAC \\
\hline N9-FIP & & 41-mer & TTCCGTTTGAGTGTTTCCCTCGAATGCAGGTTCTATGCTCT \\
\hline N9-BIP & & 39-mer & CAGTATCGCGCCCTGATAAGCCACCCAATGCATTCCACC \\
\hline N9-LB & & 23-mer & TGGCCACTATCATCACCGCCCAC \\
\hline N9-LF & & 18-mer & TGATTGTTGTTCCTTGGC \\
\hline N9 F & & 22-mer & ATAATGAAACAAACATCACCAA \\
\hline N9 R & & 22-mer & AGCATAGAACCTGCATTCATCT \\
\hline
\end{tabular}

a The primers of N9-F3, N9-B3, N9-FIP, N9-BIP, N9-LB, and N9-LF were for RT-LAMP Primers. N9-F and N9-R were applied to RT-PCR.

and a negative control (a sample devoid of template) were included in each reaction.

2.5. RT-PCR. RNA samples were amplified by RT-PCR using a one-step RNA PCR kit (Takara, Japan) with N9F and N9R primers (Table 2). The reaction conditions were set at $45^{\circ} \mathrm{C}$ for the reverse transcription for $45 \mathrm{~min}, 94^{\circ} \mathrm{C}$ for the predenaturation for $2 \mathrm{~min}$, and then 35 cycles at $94^{\circ} \mathrm{C}$ for $30 \mathrm{~s}$, $52^{\circ} \mathrm{C}$ for $45 \mathrm{~s}$, and $68^{\circ} \mathrm{C}$ for $45 \mathrm{~s}$, followed by the final extension at $68^{\circ} \mathrm{C}$ for $8 \mathrm{~min}$. The RT-PCR products were subjected to electrophoresis on a 1.5\% agarose gel, and the target bands were visualized by staining with ethidium bromide.

2.6. Sensitivity and Specificity of RT-LAMP Assay. The sensitivity of the RT-LAMP assay was tested with RNA that was extracted from serial 10-fold dilutions of H7N9 viruses. The species specificity of the H7N9 influenza virus NAspecific RT-LAMP assay was evaluated using RNA samples that were extracted from avian influenza reference N1-N9 subtype strains and other avian respiratory viral pathogens, including NDV, IBV, and ILTV. The H7N9 virus (A/chicken/Shanghai/S1053/2013(H7N9)) was used as a positive control.

2.7. Detection of Clinical Specimens. In total, 269 samples (including tracheal swabs and cloacal swabs) were collected in the experiment. Among these samples, 150 samples from chickens, 35 samples from pigeons, 30 samples from ducks, and 54 samples from the environment were collected from three live poultry markets in Shanghai in March, 2013, when the H7N9 influenza outbreak occurred there [3]. Each sample was placed in $2 \mathrm{~mL}$ minimal essential medium, which was supplemented with penicillin (2000 Units/mL) and streptomycin (2000 Units/mL). All samples were investigated using viral isolation, RT-PCR, and RT-LAMP. The virus isolation was performed in 10-day-old specific-pathogenfree embryonated chicken eggs [3]. All virus isolation procedures and RNA extractions were conducted in a biosafety level 3 facility, which was approved by the Ministry of Agriculture, China.

\section{Results}

3.1. Detection of the H7N9 Virus with the H7-RT-LAMP Assay. The H7-RT-LAMP assay was previously developed in our lab [16]. To evaluate the ability of the H7-RT-LAMP assay to detect a novel influenza A (H7N9) virus identified in humans and birds in China, H7N9 viruses that were isolated from chickens, pigeons, the environment, and humans were tested by the H7-RT-LAMP assay. All H7N9 influenza viruses can be detected in less than $30 \mathrm{~min}$ by this assay (Figure 1). The minimum detection limit of the H7-RT-LAMP assay was $0.01 \mathrm{PFU}$ per reaction for the H7N9 influenza virus that was isolated from various sources (Figures $2(\mathrm{a})-2(\mathrm{~d})$ ). These results indicated that the H7-RT-LAMP assay could be used for the detection of H7N9 influenza viruses. Because the H7RT-LAMP assay targets only the HA gene, this RT-LAMP method cannot identify the influenza virus NA subtype. To 
A/chicken/Shanghai/S1053/2013(H7N9)

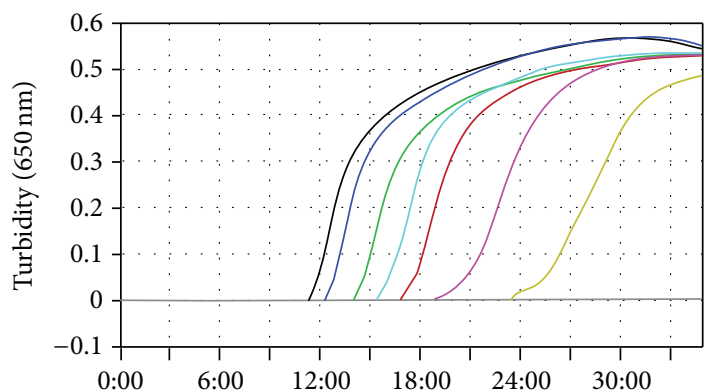

(a)

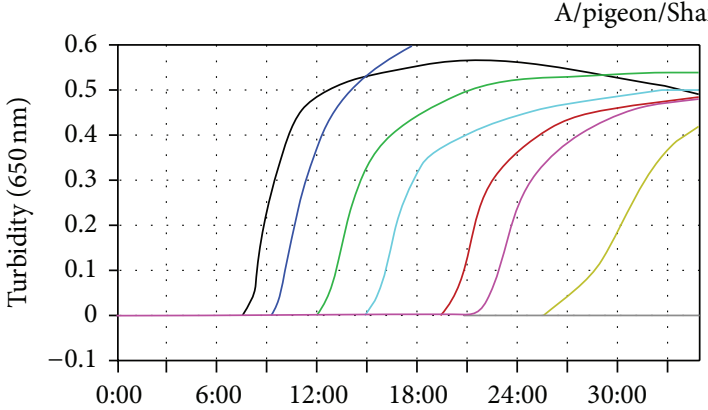

(b)

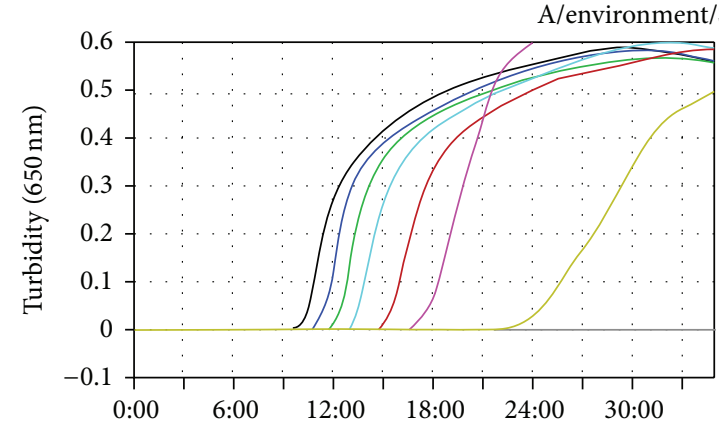

(c)
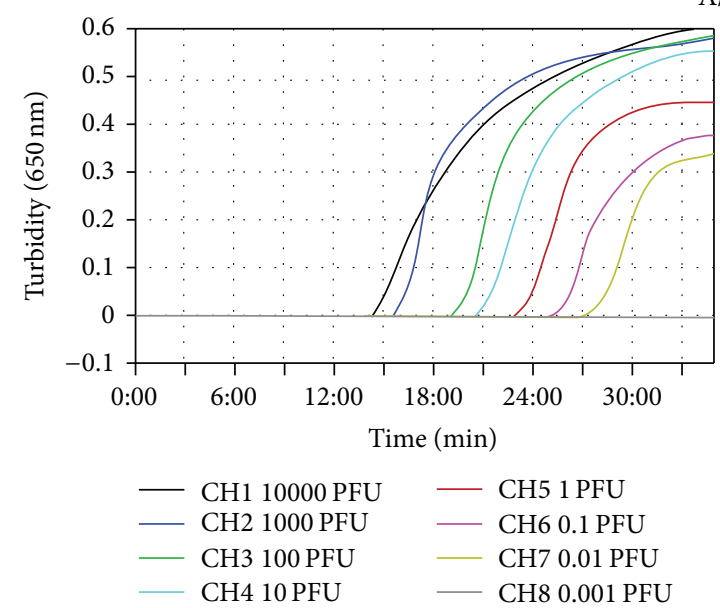

(d)

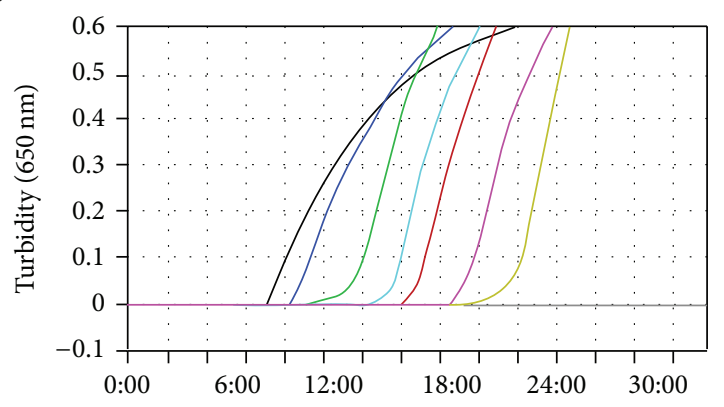

(e)

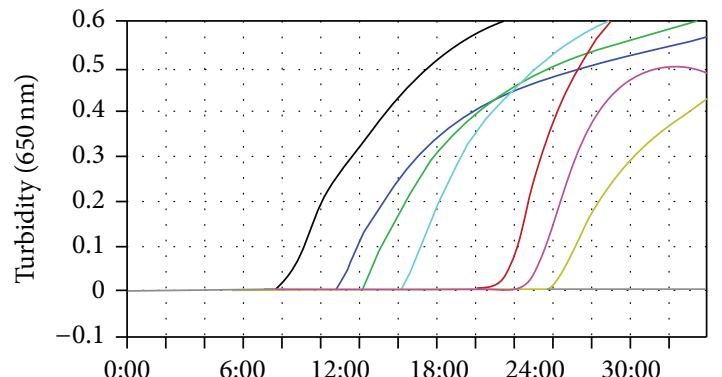

(f)

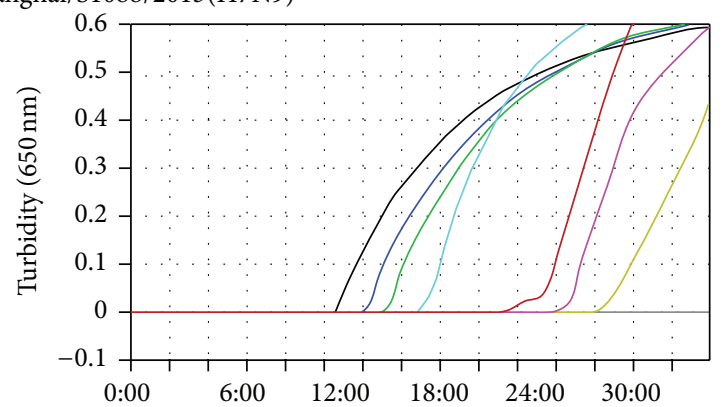

(g)

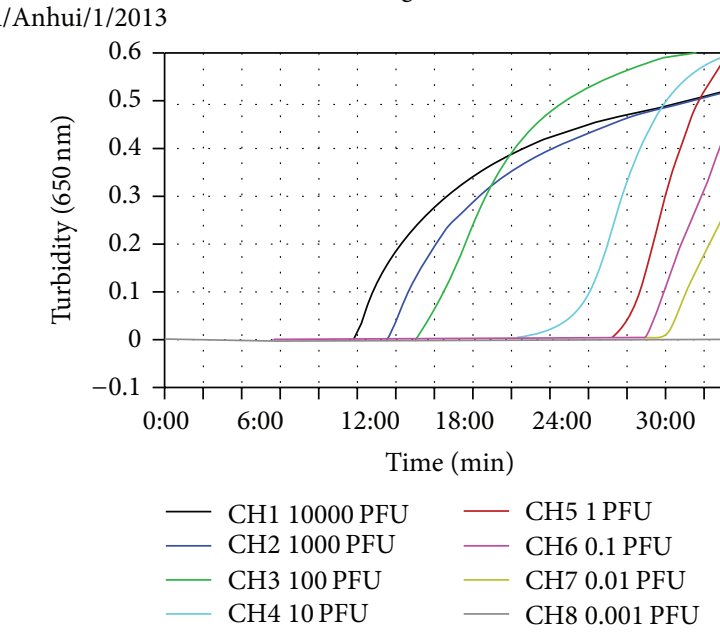

(h)

Figure 2: Sensitivity of H7-RT-LAMP and N9-RT-LAMP for the detection of H7N9 influenza viruses that were isolated from various sources. ((a) to (d)) A/chicken/Shanghai/S1053/2013 (H7N9), A/pigeon/Shanghai/S1069/2013(H7N9), A/environment/Shanghai/S1088/2013 (H7N9), and A/Anhui/1/2013 (H7N9) RNAs were amplified using H7-RT-LAMP. ((e) to (h)) A/chicken/Shanghai/S1053/2013 (H7N9), A/pigeon/Shanghai/S1069/2013 (H7N9), A/environment/Shanghai/S1088/2013 (H7N9), and A/Anhui/1/2013 (H7N9) RNAs were amplified using N9-RT-LAMP. Viral RNA concentrations ranging from 10,000 to $0.001 \mathrm{PFU}$ per tube were tested. LAMP products were detected by a real-time turbidity assay using an LA-320c. 
TABLE 3: Detection of H7N9 influenza viruses in clinical samples by virus isolation, RT-LAMP, and RT-PCR.

\begin{tabular}{|c|c|c|c|c|}
\hline \multirow{2}{*}{ Source of samples ${ }^{\mathrm{a}}$} & \multirow{2}{*}{ Total number of samples } & \multicolumn{3}{|c|}{ H7N9 virus positive samples } \\
\hline & & Virus isolation & RT-LAMP & RT-PCR \\
\hline Chickens & 142 & 10 & 10 & 10 \\
\hline Pigeons & 33 & 3 & 3 & 3 \\
\hline Ducks & 30 & 0 & 0 & 0 \\
\hline Environment $^{\mathrm{b}}$ & 54 & 5 & 5 & 3 \\
\hline Total & 259 & 18 & 18 & 16 \\
\hline
\end{tabular}

overcome this limitation, the H7N9 influenza virus NAspecific RT-LAMP (N9-RT-LAMP) assay was developed in this study.

3.2. Design of the N9-RT-LAMP Assay. The H7N9 influenza virus NA-specific RT-LAMP assay primers were designed from the alignments of the conserved sequence of the NA genes of 79 H7N9 influenza viruses (41 NA genes sequenced by our lab, 38 NA genes from GenBank), which were identified from chickens, pigeons, the environment, and humans $[1,3]$.Conserved fragments with the highest levels of homology were chosen as templates for the design of H7N9 influenza virus RT-LAMP primers. Among these 79 NA gene sequences of H7N9 influenza viruses, 3 NA gene sequences had 1 mismatched nucleotide sequence in the N9-B3 primer and 1 NA gene sequence had 1 mismatched nucleotide sequence in the N9-RT-LAMP primer. The other H7N9 influenza virus NA gene sequences matched the NA-specific RT-LAMP assay primer sequences $100 \%$. An RT-LAMP assay with the N9-specific primers was successfully developed and optimized. The optimum volumes of components in the reaction mixture included $1.6 \mathrm{mM}$ each of FIP and BIP primers, $0.2 \mathrm{mM}$ each of $\mathrm{F} 3$ and $\mathrm{B} 3$ primers, $1.2 \mathrm{mM}$ dNTPs, $8 \mathrm{mM} \mathrm{MgSO}_{4}, 0.2 \mathrm{M}$ betaine, $8 \mathrm{U}$ Bst DNA polymerase large fragment, and $1 \mu \mathrm{L}$ target DNA. The optimal temperature and time were $62.5^{\circ} \mathrm{C}$ for $30 \mathrm{~min}$.

3.3. N9-RT-LAMP Sensitivity. To evaluate the sensitivity of the N9-RT-LAMP assay with the NA primer sets, the detection limit of the assay was determined by testing against 10-fold serial dilutions of the H7N9 influenza virus (A/chicken/Shanghai/S1053/2013(H7N9)), which has a defined plaque-forming unit (PFU) dose. The kinetic analysis of the turbidity revealed that the N9-RT-LAMP assay was able to detect the H7N9 influenza virus at a level of 0.01 PFU per tube in less than 30 min (Figure 3(a)). N9-RT-LAMP sensitivity was also confirmed by observing the solution fluorescence under a UV light source. As shown in Figure 3(b), clear fluorescence signals were observed at concentrations ranging from 1000 to $0.01 \mathrm{PFU}$ per tube. There were no differences in sensitivity between the real-time turbidity and visual fluorescence detections that were associated with the LAMP assay. When the same RNA template was subjected to onestep RT-PCR using the N9-specific primers, the detection limit of the system was 1 PFU per tube (Figure 3(c)). The results indicate that the sensitivity of N9-RT-LAMP assay is approximately 100-fold higher than that of RT-PCR.

The detection limits of H7N9 influenza viruses that were isolated from various sources were determined to assess whether the N9-RT-LAMP assay is able to detect a variety of H7N9 influenza viruses at constant sensitivity. The sensitivity, which was determined by the real-time monitoring of the turbidity of H7N9 viruses that were isolated from chicken, A/chicken/Shanghai/S1053/2013 (H7N9), from pigeon, A/pigeon/Shanghai/S1069/2013 (H7N9), from human, A/Anhui/1/2013 (H7N9), and from the environment, A/environment/Shanghai/S1088/2013 (H7N9), was 0.01 PFU per tube (Figures 2(e)-2(h)). Thus, N9-RT-LAMP was adapted for the detection of H7N9 viruses that were isolated from various sources.

3.4. RT-LAMP Specificity. The N1-N9 subtype influenza viruses and three other avian respiratory viruses (NDV, IBV, and ILTV) were tested using the N9-RT-LAMP assay. Influenza virus (A/chicken/Shanghai/S1053/2013(H7N9)) was used as the positive control, and reactions that were performed in the absence of the template were used as negative controls. Only the H7N9 influenza virus was positive, and no LAMP products were detected in the reactions that were performed with RNA that was harvested from avian influenza viruses of different subtypes (Figure 4(a)) or from RNAs that were collected from other avian respiratory viruses (Figure 4(b)). These results demonstrated that the N9-RT-LAMP assay was specific and could be used to specifically detect the N9 subtype influenza virus.

3.5. Evaluation of the N9-RT-LAMP Assay Using Clinical Samples. To evaluate the ability of the N9-RT-LAMP assay to detect H7N9 viruses from clinical samples, 259 clinical samples were collected from three live poultry markets in Shanghai. All samples were tested by RTLAMP and RT-PCR. Of the 259 samples that were evaluated, N9-RT-LAMP gave 18 positive cases, whereas RT-PCR gave 16 positive cases (Table 3 ). The positive rates after RT-LAMP and RT-PCR were 6.9\% (18/259) and $6.2 \%(16 / 259)$, respectively. H7N9 viruses were isolated from 18 of the 18 positive samples by RT-LAMP. The results 


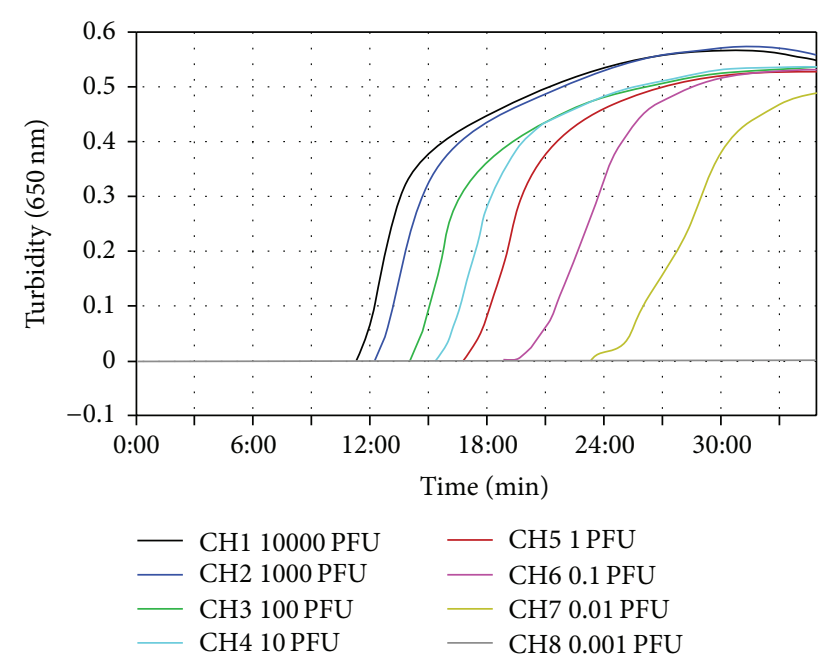

(a)

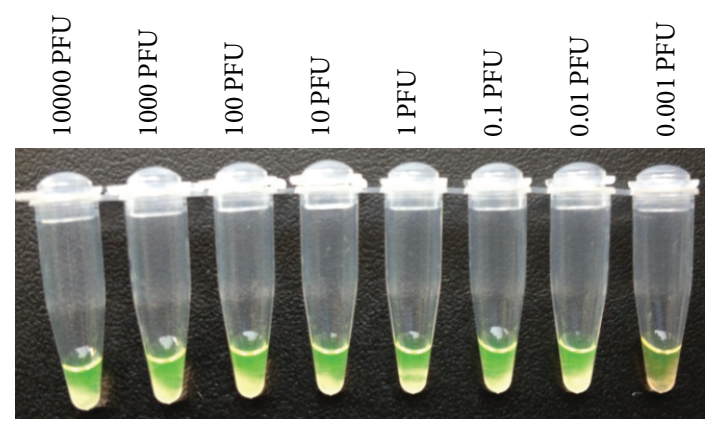

(b)

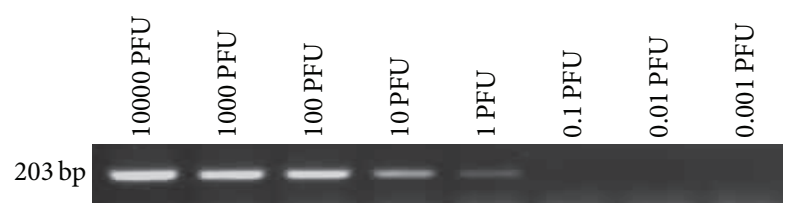

(c)

FIGURE 3: Comparative sensitivity of the RT-LAMP and RTPCR methods. N9-RT LAMP and RT-PCR were performed using A/Shanghai/4664T/2013 (H7N9) viral RNA at concentrations ranging from 10,000 to 0.001 PFU per tube. ((a) and (b)) Detection limit of N9-RT-LAMP. LAMP products were detected using a real-time turbidity assay with an LA-320c (a) and a fluorescence assay (b). (c) Detection limit for the one-step RT-PCR using the same RNA extracts that were used for N9-RT LAMP. The PCR products were observed in a $1.5 \%$ agarose gel that was stained with ethidium bromide.

indicated that two N9-RT-LAMP-positive specimens were missed by RT-PCR.

\section{Discussion}

The novel influenza H7N9 virus has emerged and raises serious concerns for public health $[1,17]$. No vaccine for the prevention of avian influenza A (H7N9) infections in humans and animals is currently available [18]. At present, the control of H7N9 virus infections primarily depends on the early identification and the quarantine of infected cases to prevent

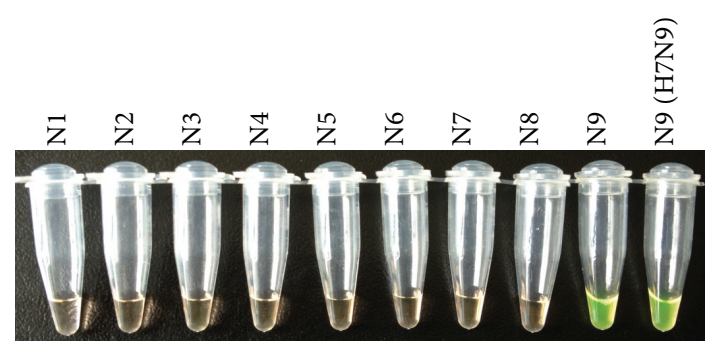

(a)

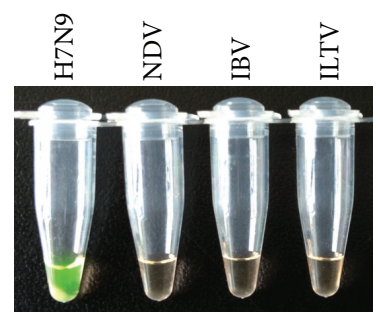

(b)

FIGURE 4: Specificity of the N9-RT-LAMP assay for different subtype influenza viruses and other avian respiratory viruses. Viral RNAs were extracted from the representative influenza viruses of avian origin (subtypes N1-9) (a) and other avian respiratory viruses (b). The RT-LAMP reaction was assessed based on the fluorescence intensity. N1, A/Goose/Guangdong/1/96 (H5N1); N2, A/Turkey/Wisconsin/1/66 (H9N2); N3, A/Duck/Germany/1215/73 (H2N3); N4, A/Turkey/Ontario/6118/68 (H8N4); N5, A/Mallard/ Gurjev/263/82 (H14N5); N6, A/Duck/Czech/56 (H4N6); N7, A/equine/jingfang/1/74 (H7N7); N8, A/Duck/Ukraine/1/63 (H3N8); N9, A/Duck/Memphis/546/76 (H11N9); N9 (H7N9), A/chicken/ Shanghai/S1053/2013;

the further spread of the virus. Therefore, the development of a simple and rapid diagnostic method for this H7N9 virus is extremely important. In this study, specific, sensitive, and quick RT-LAMP methods were developed for H7N9 virus detection. RT-LAMP methods can detect different sources of the H7N9 virus (isolated from humans, chickens, pigeons, and the environment) in $30 \mathrm{~min}$.

The H7-RT-LAMP assay was previously developed based on the HA genes in our lab [16]. We evaluated the ability of the H7-RT-LAMP assay to detect different sources of H7N9 viruses. The results indicated that the H7-RT-LAMP assay could detect as low as 0.01 PFU of different sources of H7N9 virus. This observation is consistent with the sensitivity of the assay for the detection of LPAI-H7N2 [16]. We compared the H7-RT-LAMP primer sequences and target sequences of the H7N9 virus. There are two nucleotide mismatches in the H7-RT-LAMP primer between H7N9 influenza viruses. The results of sensitivity indicated that the two nucleotide mismatches did not influence the amplification efficiency of the H7-RT-LAMP primer for the detection of the H7N9 virus.

We investigated the utility of NA-specific RT-LAMP primer sets for the rapid and accurate detection of different sources of H7N9 influenza virus RNA (from chickens, pigeons, the environment, and humans). The N9-RT-LAMP assay detected different sources of H7N9 influenza virus with 
a detection limit of 0.01 PFU (Figures 2 and 3). When the sensitivity of N9-RT-LAMP was compared with that of one-step RT-PCR using an H7N9 influenza virus dilution series, N9RT-LAMP was approximately 100 -fold more sensitive than RT-PCR. Two clinical samples tested negative by the RT-PCR but tested positive by the N9-RT-LAMP assay and by virus isolation. The results further indicated that the H7N9 virus RT-LAMP assay was slightly more sensitive than the RT-PCR for the detection of the H7N9 virus NA gene in specimens.

In addition, there is no cross-reactivity with other NA subtype influenza virus RNAs or other avian respiratory virus RNAs, suggesting that H7N9 virus NA-specific RT-LAMP has high specificity among some common avian viruses at the RNA level.

Since April 2013, H7N9 viruses that are similar to those viruses that were isolated from patients have been isolated from pigeons and chickens $[1,3]$. The viruses can efficiently transmit among poultry, particularly chickens, and have spread from live poultry markets in Shanghai to eight other provinces in a relatively short period $[4,19,20]$. The viruses are nonpathogenic to poultry, which enable the avian H7N9 virus to replicate silently in avian species, and are severe threat to human health [4]. Thus, the timely surveillance of samples that are involved in poultry is extremely important for monitoring the prevalence of the H7N9 virus [21-23]. To evaluate the practicability of H7N9 virus RT-LAMP assays for the detection of avian-origin samples in this study, many clinical samples (including drinking water, soil, cloacal swab, and tracheal swab samples) that were collected from live poultry markets, poultry farms, and wild bird habitats from Shanghai and Anhui were tested by the H7N9 virus RTLAMP assays. The results of the H7N9 virus RT-LAMP assays were consistent with those of the virus isolation [3]. Although 30 min was used for the H7N9 RT-LAMP reactions, most of the amplification reactions for clinical samples could be finished within $25 \mathrm{~min}$. These results suggested that the H7N9 virus RT-LAMP assays were efficient, practical, and rapid diagnostic methods for the detection of the influenza A (H7N9) virus from different sources.

In summary, H7N9 virus RT-LAMP assays were developed and validated in this study for the detection of the H7N9 influenza virus with high sensitivity and specificity. The RT-LAMP assays required minimal laboratory equipment and could detect different sources of H7N9 influenza viruses. Moreover, compared with the requirements that are associated with RT-PCR and with virus isolation procedures, H7N9 virus RT-LAMP assays were significantly more rapid. Obtaining results within $2 \mathrm{~h}$ (including the extraction of RNA) is important for the rapid detection of the H7N9 influenza virus infection; thus, the present methods may be useful for the rapid detection of humans or animals that have been infected by the H7N9 influenza virus. Although FAO recommended that the diagnostic method for the H7N9 virus was the real-time RT-PCR (rRT-PCR) assay (http://www.who.int/influenza/gisrs_laboratory/cnic_realtime_rt_pcr_protocol_a_h7n9.pdf) [24], rRT-PCR requires an expensive machine system and experienced laboratory workers. We hope that this new, effective, and rapid diagnostic method will contribute to the control of the H7N9 influenza virus infection.

\section{Conclusion}

The specific, sensitive, and quick RT-LAMP methods were developed for H7N9 influenza virus detection. The RTLAMP methods can detect the H7N9 virus from different resource samples (isolated from humans, chickens, pigeons, and the environment) in less than 30 min with high sensitivity and specificity. The RT-LAMP methods will contribute to the control of the H7N9 influenza virus infection.

\section{Conflict of Interests}

The authors declare that there is no conflict of interests regarding the publication of this paper.

\section{Acknowledgments}

This study was supported by Key Special Fund for Agroscientific Research in the Public Interest (200903055) and the 12th five-year plan of national science and technology for the rural areas (2012AA101303).

\section{References}

[1] R. Gao, B. Cao, Y. Hu et al., "Human infection with a novel avian-origin influenza A (H7N9) virus," The New England Journal of Medicine, vol. 368, no. 20, pp. 1888-1897, 2013.

[2] World Health Organisation, "Number of confirmed human cases of avian influenza A(H7N9) reported to WHO," 2013, http://www.who.int/influenza/human_animal_interface/influenza_h7n9/09_ReportWebH7N9Number.pdf.

[3] J. Shi, G. H. Deng, P. H. Liu et al., "Isolation and characterization of H7N9 viruses from live poultry markets-implication of the source of current H7N9 infection in humans," Chinese Science Bulletin, vol. 58, no. 16, pp. 1857-1863, 2013.

[4] Q. Zhang, J. Shi, G. Deng et al., "H7N9 influenza viruses are transmissible in ferrets by respiratory droplet," Science, vol. 341, no. 6144, pp. 410-414, 2013.

[5] T. Kubo, M. Agoh, L. Q. Mai et al., "Development of a reverse transcription-loop-mediated isothermal amplification assay for detection of pandemic (H1N1) 2009 virus as a novel molecular method for diagnosis of pandemic influenza in resource-limited settings," Journal of Clinical Microbiology, vol. 48, no. 3, pp. 728$735,2010$.

[6] V. M. Corman, M. Eickmann, O. Landt et al., "Specific detection by real-time reverse-transcription PCR assays of a novel avian influenza $\mathrm{A}(\mathrm{H} 7 \mathrm{~N} 9)$ strain associated with human spillover infections in China," Eurosurveillance, vol. 18, no. 16, article 2, Article ID 20461, 2013.

[7] C. K. Wong, H. Zhu, O. T. Li et al., "Molecular detection of human H7N9 influenza A virus causing outbreaks in China," Clinical Chemistry, vol. 59, no. 7, pp. 1062-1067, 2013.

[8] C. Qiu, A. Zhang, D. Tian et al., "Safe pseudovirus-based assay for neutralization antibodies against influenza A(H7N9) virus," Emerging Infectious Disease, vol. 19, no. 10, pp. 1685-1686, 2013.

[9] K. Nie, X. Zhao, X. Ding et al., "Visual detection of human infection with influenza A (H7N9) virus by subtype-specific 
reverse transcription loop-mediated isothermal amplification with hydroxynaphthol blue dye," Clinical Microbiology and Infection, vol. 19, no. 8, pp. E372-E375, 2013.

[10] W. Xu, L. Lu, B. Shen, J. Li, J. Xu, and S. Jiang, "Serological investigation of subclinical influenza A(H7N9) infection among healthcare and non-healthcare workers in Zhejiang Province, China," Clinical Infectious Diseases, vol. 57, no. 6, pp. 919-921, 2013.

[11] T. Bai, J. F. Zhou, and Y. L. Shu, "Serologic study for influenza A (H7N9) among high-risk groups in China," The New England Journal of Medicine, vol. 368, no. 24, pp. 2339-2340, 2013.

[12] E. Chen, F. Wang, H. Lv et al., "The first avian influenza A (H7N9) viral infection in humans in Zhejiang Province, China: a death report," Frontiers of Medicine, vol. 7, no. 3, pp. 333-344, 2013.

[13] T. Notomi, H. Okayama, H. Masubuchi et al., "Loop-mediated isothermal amplification of DNA," Nucleic Acids Research, vol. 28, no. 12, article e63, 2000.

[14] M. Imai, A. Ninomiya, H. Minekawa et al., "Rapid diagnosis of $\mathrm{H} 5 \mathrm{~N} 1$ avian influenza virus infection by newly developed influenza $\mathrm{H} 5$ hemagglutinin gene-specific loop-mediated isothermal amplification method," Journal of Virological Methods, vol. 141, no. 2, pp. 173-180, 2007.

[15] Y. Mori, K. Nagamine, N. Tomita, and T. Notomi, "Detection of loop-mediated isothermal amplification reaction by turbidity derived from magnesium pyrophosphate formation," Biochemical and Biophysical Research Communications, vol. 289, no. 1, pp. 150-154, 2001.

[16] H. Bao, X. Wang, Y. Zhao et al., "Development of a reverse transcription loop-mediated isothermal amplification method for the rapid detection of avian influenza virus subtype H7," Journal of Virological Methods, vol. 179, no. 1, pp. 33-37, 2012.

[17] Y. Chen, W. Liang, S. Yang et al., "Human infections with the emerging avian influenza A H7N9 virus from wet market poultry: clinical analysis and characterisation of viral genome," The Lancet, vol. 381, no. 9881, pp. 1916-1925, 2013.

[18] M. T. Osterholm, K. S. Ballering, and N. S. Kelley, "Major challenges in providing an effective and timely pandemic vaccine for influenza A(H7N9)," Journal of the American Medical Association, vol. 309, no. 24, pp. 2557-2558, 2013.

[19] C. Xu, F. Havers, L. Wang et al., "Monitoring avian influenza A(H7N9) virus through national influenza-like illness surveillance, China," Emerging Infectious Disease, vol. 19, no. 8, pp. 1289-1292, 2013.

[20] R. B. Tang and H. L. Chen, "An overview of the recent outbreaks of the avian-origin influenza A (H7N9) virus in the human," Journal of the Chinese Medical Association, vol. 76, no. 5, pp. 245-248, 2013.

[21] J. Han, M. Jin, P. Zhang et al., "Epidemiological link between exposure to poultry and all influenza A(H7N9) confirmed cases in Huzhou city, China, March to May 2013," Eurosurveillance, vol. 18, no. 20, article 2, Article ID 20481, 2013.

[22] C.-J. Bao, L.-B. Cui, M.-H. Zhou, L. Hong, G. F. Gao, and H. Wang, "Live-animal markets and influenza A (H7N9) virus infection," The New England Journal of Medicine, vol. 368, no. 24, pp. 2337-2339, 2013.

[23] C. Wang, J. Wang, W. Su et al., "Relationship between domestic and wild birds in live poultry market and a novel human H7N9 virus in China," The Journal of Infectious Disease, 2013.

[24] WHO, "Human infection with influenza A(H7N9) virus in China-update," 2013, http://www.who.int/csr/don/2013_05 _02/en/index.html. 

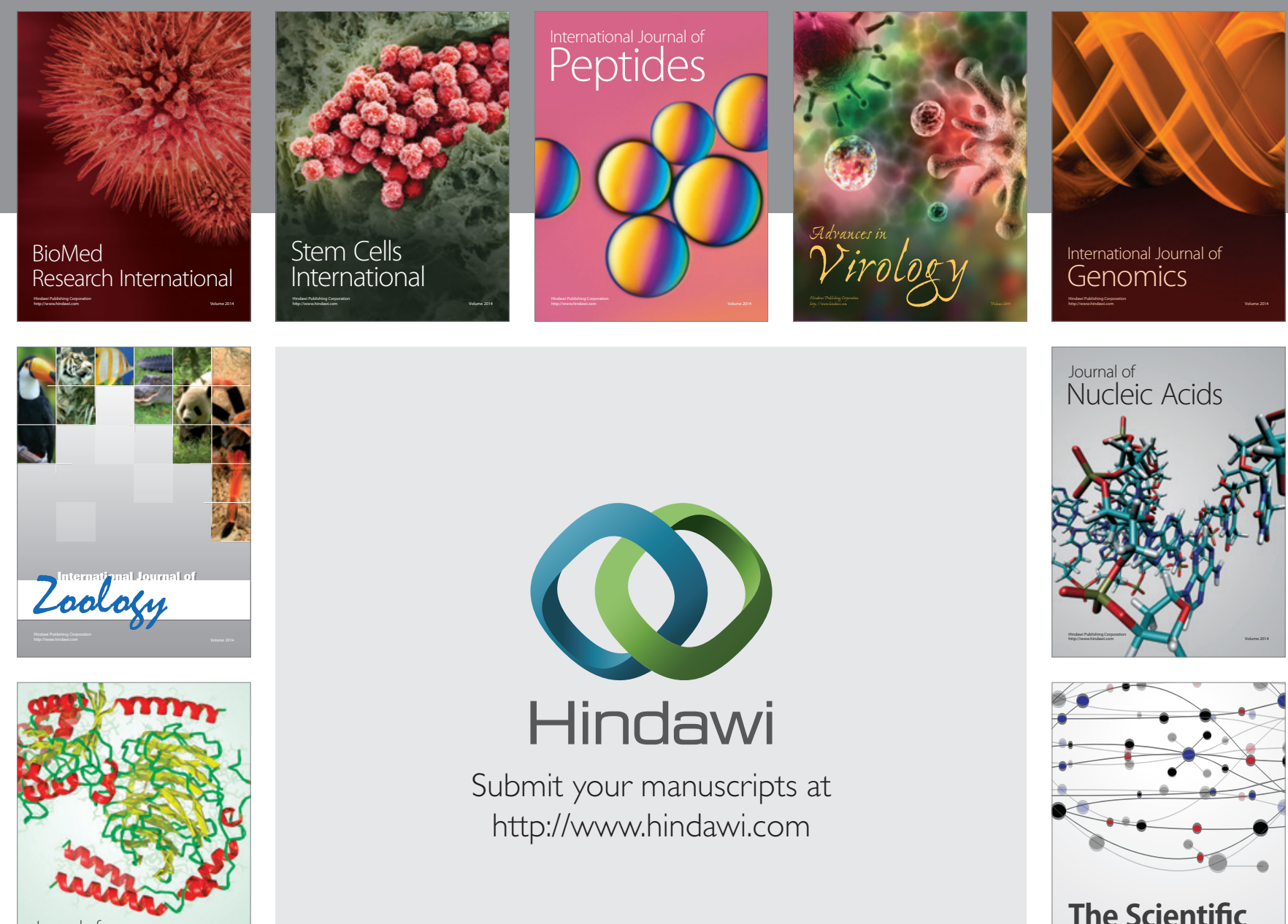

Submit your manuscripts at

http://www.hindawi.com

Journal of
Signal Transduction
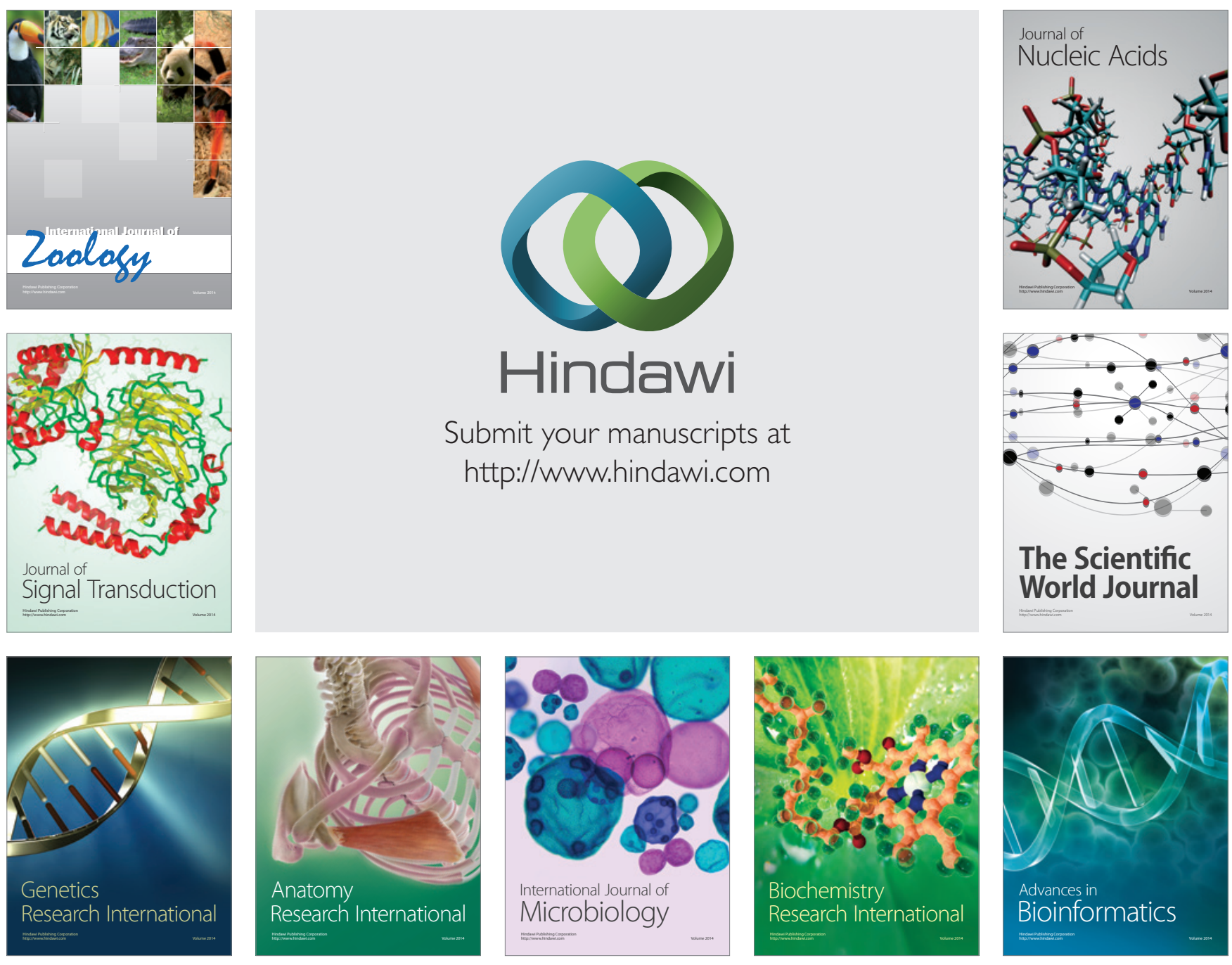

The Scientific World Journal
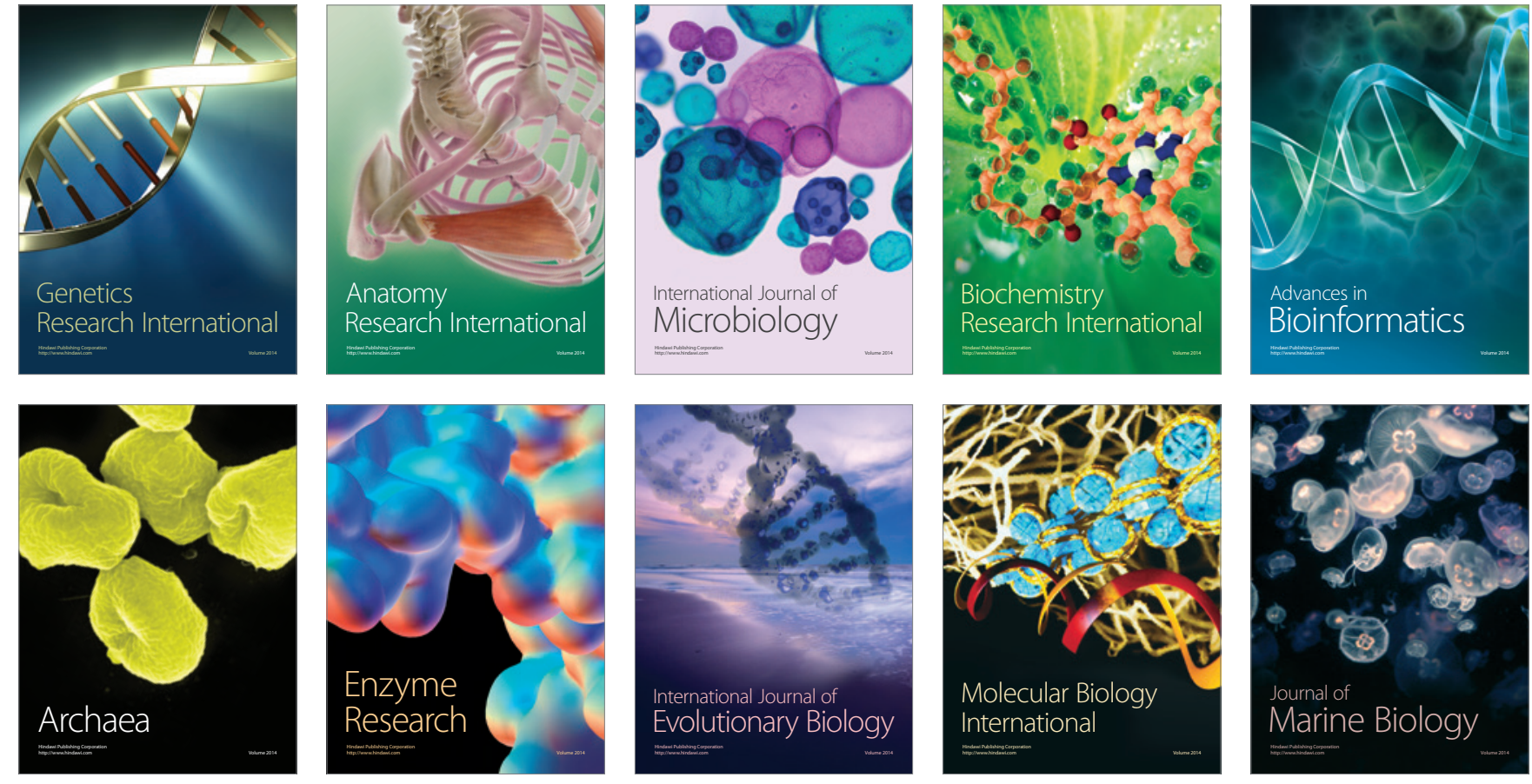